\title{
Cardiotoxicity mechanisms of the combination of BRAF-inhibitors and MEK-inhibitors
}

\author{
Enrico Bronte ${ }^{\mathrm{a}, *, 1}$, Giuseppe Bronte ${ }^{\mathrm{b}, 1}$, Giuseppina Novo ${ }^{\mathrm{c}}$, Gaetana Rinaldi ${ }^{\mathrm{a}}$, Fabrizio Bronte ${ }^{\mathrm{d}}$, \\ Francesco Passiglia ${ }^{\mathrm{a}}$, Antonio Russo ${ }^{\mathrm{a}}$
}

a Department of Surgical, Oncological and Oral Sciences, Section of Medical Oncology, University of Palermo, Palermo, Italy

b Department of Medical Oncology, Istituto Scientifico Romagnolo per lo Studio e la Cura dei Tumori (IRST) IRCCS, Meldola, Italy

c Division of Cardiology, Department of Internal Medicine and Specialties, University of Palermo, Palermo, Italy

${ }^{\mathrm{d}}$ U.O.C. di Gastroenterologia, Ospedali Riuniti Villa Sofia-Cervello, Palermo, Italy

\section{A R T I C L E I N F O}

\section{Keywords:}

BRAF inhibitor

MEK inhibitor

Cardiovascular toxicity

Cardio-oncology

Hypertension

Decreased ejection fraction

\begin{abstract}
A B S T R A C T
Many new drugs have appeared in last years in the oncological treatment scenario. Each drug carries an important set of adverse events, not less, cardiovascular adverse events. This aspect is even more important considering the increasing use of combination therapies with two drugs, or three drugs as in some ongoing clinical trials. Besides it represents a growing problem for Cardiologists, that face it in every day clinical practice and that will face it probably more and more in the coming years. This work reviews the mechanism of action of BRAF-inhibitors and MEKinhibitors used together, the pathophysiological mechanisms that lead to cardiovascular toxicity. Particularly, it focuses on hypertension and ejection fraction reduction development. Then, it follows the examination of published data for each combination therapy. A Literature research was carried out using Pubmed selecting review articles, original studies and clinical trials, but mainly focusing on phase 3 studies. This work aims to summarize the knowledge about BRAF-inhibitor and MEK-inhibitor treatment and its cardiovascular toxicity to make it usable and give the basic tools to Cardiologists and Oncologists for a better management of cancer patient undergoing this treatment. Besides a deeper knowledge of the cardiovascular adverse events linked to this treatment and the magnitude of their expression and frequency can lead to a targeted cardiological treatment.
\end{abstract}

(c) 2018 Elsevier Inc. All rights reserved.

\section{Contents}

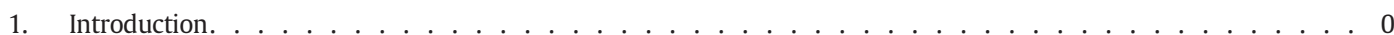

2. Mechanism of action of BRAF-inhibitor and MEK-inhibitor combined . . . . . . . . . . . . . . . . . . . . . . 0

3. Pathophysiological mechanisms of cardiovascular toxicity of BRAF-inhibitor plus MEK-inhibitor . . . . . . . . . . 0

4. Cardiovascular toxicity by BRAF-inhibitor plus MEK-inhibitor . . . . . . . . . . . . . . . . 0

5. Perspectives of combination therapy. . . . . . . . . . . . . . . . . . . . . . . . . . 0

$6 . \quad$ Discussion and conclusions . . . . . . . . . . . . . . . . . . . . . . . . . 0

Conflicts of interest statement $\ldots . \cdots \cdot 0$

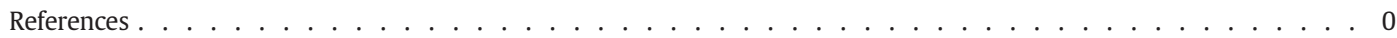

Abbreviations: 6MHP, Melanoma Helper Peptide Vaccine; BRAFi, BRAF inhibitors; CD47, Cluster of Differentiation 47; cGMP, cyclic guanosine monophosphate; CRC, colorectal cancer;

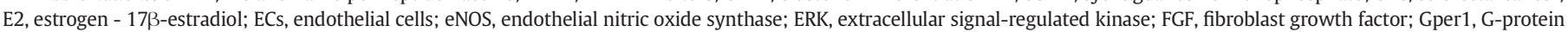

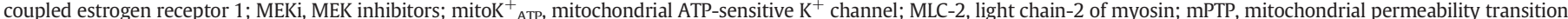

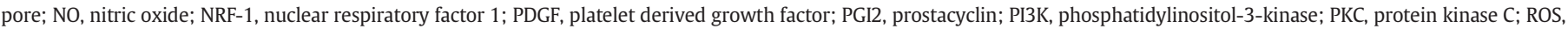

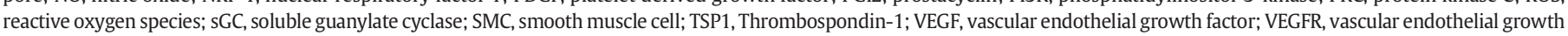
factor receptor; VSMCs, vascular smooth muscle cells.

* Corresponding author at: Department of Surgical, Oncological and Oral Sciences, Section of Medical Oncology, University of Palermo, Via del Vespro n. 129, 90127 Palermo, Italy.

E-mail address: enrico.bronte@gmail.com (E. Bronte).

1 These authors contributed equally to this work. 


\section{Introduction}

The increasing observation of cardiovascular toxicity by anti-cancer drugs during the last decades led to the development of a new field of study, which is Cardio-Oncology. This is linked to the fact that the epidemiological increase of cancer patients in the last decade has led researchers to find continuously better therapies in the fight against cancer. The earliest evidence of cardiovascular toxicity concerned chemotherapy, especially anthracycline, and radiotherapy. (Cardinale et al., 2015; Di Lisi et al., 2017; Lahtinen, Kuikka, Nousiainen, Uusitupa, \& Länsimies, 1991) Then several data about cardiovascular toxicity emerged from clinical trials on monoclonal antibodies, such as trastuzumab. Later, other targeted therapies were developed, which showed likewise cardiovascular toxicity, particularly tyrosine kinase inhibitors targeting vascular endothelial growth factor receptor (VEGFR) (Bronte, Bronte, Novo, Bronte, et al., 2015; Bronte, Bronte, Novo, Pernice, et al., 2015). BRAF inhibitors (BRAFi) cardiovascular toxicity has emerged lately as a critical safety issue for the important use of this class of drugs in the treatment of melanoma. (Bronte, Bronte, Novo, Bronte, et al., 2015; Bronte, Bronte, Novo, Pernice, et al., 2015) Unfortunately, the use of BRAFi has highlighted the problem of the onset of treatment-related resistance. For this reason, MEK inhibitors (MEKi) have been developed and studied (Banks, Crowell, Proctor, \& Jensen, 2017; Flaherty, Infante, et al., 2012; Flaherty, Robert, et al., 2012), showing nevertheless better results on disease when used in combination, BRAFi plus MEKi (Banks et al., 2017; Flaherty, Infante, et al., 2012; Flaherty, Robert, et al., 2012). It has to be noted that each of these drugs showed a cardiovascular toxicity profile. In fact, on one hand BRAFi have mainly drawn attention to hypertension and QT interval (QT) prolongation at ECG (Bronte, Bronte, Novo, Bronte, et al., 2015; Bronte, Bronte, Novo, Pernice, et al., 2015), on the other hand cardiovascular adverse events such as peripheral oedema, hypertension, decreased cardiac ejection fraction, have been usually linked to MEKi treatment (Banks et al., 2017). These last adverse events are more frequent using the combination therapy than the monotherapy, even though they are readily reversible when the drug is interrupted (Banks et al., 2017; Dossett, Kudchadkar, \& Zager, 2015; Flaherty, Infante, et al., 2012; Flaherty, Robert, et al., 2012).

In the present work, we aim to make a review of Literature to highlight the magnitude of the problem of cardiovascular toxicity due to the use of combination therapy BRAFi plus MEKi. We start from the mechanism of action of this treatment, passing through the pathophysiological mechanisms underlying the onset of cardiovascular toxicity linked to the treatment. Then, we examine the cardiovascular toxicity data related to the three combination treatments studied to date, Dabrafenib plus Trametinib, Vemurafenib plus Cobimetinib, Encorafenib plus Binimetinib based on clinical trials. Finally, we summarize the future perspectives of the combination treatments and the implications that these have on clinical practice.

\section{Mechanism of action of BRAF-inhibitor and MEK-inhibitor combined}

Melanoma is an aggressive disease that in past years was fatal in a short lapse of time. The increasing incidence led to understand the molecular basis of this disease. Besides, the lack of therapies in melanoma has led to an explosion of research in this area of interest. Research highlighted the key role of RAS-RAF-pathway (mitogenactivated protein-MAP-kinase) in various cancer, primarily melanoma. RAS is the first mediator of the pathway, it changes state from the active form tied to GTP to the inactive form tied to GDP. There are various isoforms of RAS, which are H-RAS, N-RAS and K-RAS. Activated RAS starts a signaling cascade that carries important mitogenic signals from the cell surface to the nucleus. In fact, at cell surface, RAS is activated by the interaction of growth factors with their respective receptors. It powers up in turn several signaling pathways, among which two have been studied extensively (Bronte, Bronte, Novo, Bronte, et al., 2015; Bronte, Bronte, Novo, Pernice, et al., 2015; Khan et al., 2018), which are the RAF/MEK/extracellular signal-regulated kinase (ERK) pathway and the phosphatidylinositol-3-kinase (PI3K)/PTEN/ Akt pathway. Besides, these two pathways can cross-talk. So, activated RAS phosphorylates RAF in its isoforms, ARAF, BRAF, and CRAF/Raf- 1 . Then RAF isoforms activate the MEK kinase (1/2), phosphorylating it, which in turn triggers ERK kinase (1/2). ERK promotes the transcription of important genes for cell proliferation and differentiation and resistance to apoptosis (Bronte, Bronte, Novo, Bronte, et al., 2015; Bronte, Bronte, Novo, Pernice, et al., 2015). Therefore, the presence of a mutation at any level in the pathway results in a constitutive activation of MAPK signaling pathway promoting and maintaining tumor development and growth. Each molecule in the cascade of MAP-kinase pathway, which are RAS-RAF-MEK-ERK, could be hit by a mutation. For this reason, researchers identified the mediators of this pathway as a therapeutic target for intervention. The first mediator studied was RAF, in particular its isoform BRAF. Studies demonstrated that it is mutated in melanoma in about $50-60 \%$ of cases. Besides, the most common mutation is the substitution of a valine to glutamine at codon 600 in exon 15 , V600E, that has been found in about $90 \%$ of BRAF-mutant melanomas. Other mutations are V600 K, V600D or V600R. (Alsina et al., 2003; Koo et al., 2002) The identification of the BRAF mutation has led to the development of this new class of targeted drugs, which selectively inhibits BRAF. The first drug developed was Vemurafenib, then it was developed Dabrafenib and more recently Encorafenib. (Bronte, Bronte, Novo, Bronte, et al., 2015; Bronte, Bronte, Novo, Pernice, et al., 2015; Cartlidge et al., 2008; Davies et al., 2002; Hauschild et al., 2012; Li et al., 2016; McArthur et al., 2014).

The selective inhibition of BRAF causes the inhibition of the hyperactivation of the pathway, thus limiting the excessive cell proliferation and balancing proliferation and apoptosis. The experience gained with these drugs, however, showed not only the development of treatment-related resistance, but also a paradoxical hyperactivation of the signaling cascade mediated by MEK. The development of molecules directed to the inhibition of MEK aimed to suppress this hyperactivation. So, the double block of BRAF and MEK, resulting in the control of cell proliferation, is a successful strategy in the management of the disease (Bronte, Bronte, Novo, Bronte, et al., 2015; Bronte, Bronte, Novo, Pernice, et al., 2015; Collisson, De, Suzuki, Gambhir, \& Kolodney, 2003; Kaplan, Shao, Mayberry, \& Aplin, 2011; Sumimoto, Imabayashi, Iwata, \& Kawakami, 2006).

\section{Pathophysiological mechanisms of cardiovascular toxicity of BRAF-inhibitor plus MEK-inhibitor}

Some studies highlighted in animal models that vascular endothelial growth factor (VEGF) has a protective action on vasculature because perivascular VEGF gene transfer induces the inhibition of neointimal smooth muscle cell (SMC) hyperplasia without angiogenesis. The nitric oxide (NO) pathway is involved in this process. In fact, many studies showed that VEGF stimulates endothelial production of NO and prostacyclin (PGI2). These mediators have a vascular protective effect because they are involved in vasodilation, antiproliferative effects in SMCs and anti-platelet actions. Besides, NO causes the inhibition of leukocyte interactions with endothelium, thus inhibiting leukocyte rolling and adhesion and upregulation of intercellular adhesion molecule- 1 and vascular cell adhesion molecule-1. Both NO and PGI2 mediate angiogenic and permeability-increasing effects of VEGF. Adhesion molecule expression and leukocyte adhesion showed an important role in the early stages of atherosclerosis, so VEGF-induced NO synthesis could result in an anti-inflammatory effect, which follows a protection against proatherogenic factors. The production of NO and PGI2 induced by VEGF is mediated by ERK1/2-dependent activation of cytosolic phospholipase A2 and protein kinase C (PKC). RAF isoforms, so, are stimulated by PKC and independently by RAS, which is induced by VEGF/VEGFR 
interaction. The activation of RAF isoforms then stimulated the MEK/ ERK downstream. (Bronte, Bronte, Novo, Bronte, et al., 2015; Bronte, Bronte, Novo, Pernice, et al., 2015; Hausenloy \& Yellon, 2009; Zachary, 2001).

Patients with hypertrophic cardiomyopathy showed an expression of Ras mRNA, which was positively correlated with the severity of hypertrophy. Besides, patients affected by the so-called RAS/MAPK syndromes, which are a group of autosomal dominant disorders linked to mutations causing increased RAS/RAF/MEK/ERK activity, such as Noonan and Leopard syndromes, have hypertrophic cardiomyopathy. Besides, in vitro and in vivo studies showed that constitutive active MEK1 induces cardiomyocyte hypertrophy, while on the contrary dominant negative MEK1 decreases this response. Even though MEK1 transgenic heart did not show an increase in fibrosis and had preserved cardiac function, suggesting that the MEK-ERK downstream is not the only one involved for RAS-induced pathological remodeling. (Rose, Force, \& Wang, 2010).

The activation of MEK 1/2 in cardiomyocytes provokes on one hand cardioprotection after myocardial infarction, on the other hand its chronic activation causes, in mice, dilated cardiomyopathy. The cardiac adverse events are directly related to the suppression of ERK1/2 activation in the heart. So, it is not due to an off-target effect. Futhermore, the MEK-ERK1/2 signaling plays an important role in the regulation of the renin angiotensin system toward the sympathetic nervous system, in the brain of heart failure rats. (Kubin et al., 2011; Zhu et al., 2017)

The first most important adverse event linked to the combination therapy is hypertension. In order to understand the underlying mechanism, it is important to examine various molecules that are involved in the process. Cluster of Differentiation 47 (CD47) is a transmembrane protein. It is expressed in several tissues as part of the self. Studies evaluated the possible action that BRAF and MEK inhibition can exert on CD47 and its expression in melanoma cells. The use of BRAF and MEK inhibitors upregulates the expression of $\mathrm{CD} 47$ on cell surface in melanoma cells both in vitro and in vivo. This is due to a transcriptional increase of
CD47 level, which was also linked to a rebound activation of ERK, suggesting an involvement of ERK reactivation. In fact, the activation of the transcriptional fragment of the CD47 promoter was due to ERK action. Besides, there is evidence that knockdown models of ERK1/2 by siRNA induced a reduction of CD47. This increase in CD47 levels is mediated by the transcription factor, nuclear respiratory factor 1 (NRF-1). NRF-1 has a regulatory role in the transcription of nuclearencoded mitochondrial genes, so it connects the coordination between nuclear and mitochondrial genomes. Its activation is subsequent to phosphorylation and relocation from the cytoplasm to the nucleus. (Liu et al., 2017)

Studies aiming to understand the role of Thrombospondin-1 (TSP1) have helped in understanding the role played by CD47. TSP1 is a large, secreted glycoprotein. It is secreted by platelets when they are activated. It is one of the major components of platelet $\alpha$-granules. So, it has an important role in thrombosis and hemostasis. TSP-1 on its $\mathrm{N}$-terminal domain interacts with various receptors on cell surface, while on the G-terminal domain interacts functionally with CD47. TSP-1 is an endogenous anti-angiogenic molecule, which counterbalances tumor-driven angiogenesis and regulates episodes of angiogenesis in the adult. This activity involves the regulation of NO signaling. In vivo models studies demonstrated that TSP- 1 through the action of CD47 inhibits the NO stimulation of soluble guanylate cyclase (sGC) in both endothelial cells (ECs) and vascular smooth muscle cells (VSMCs). So, CD47 is an important mediator of transition in this pathway. It inhibits $\mathrm{NO} /$ cyclic guanosine monophosphate (cGMP) signaling. It also inhibits the direct activation of cGK by cGMP analogs. NO is a molecule that has a remarkable importance in regulating the cardiovascular system and its homeostasis (Fig. 1). In fact, it regulates blood pressure and vascular tone. It also has a role in regulating tissue perfusion when metabolic needs increase. Shear stress and the increase in calcium levels activate endothelial nitric oxide synthase (eNOS), that produce NO. This last one then spreads going through membranes into VSMC, activating sGC. sGC produces cGMP, which causes a cGK-dependent

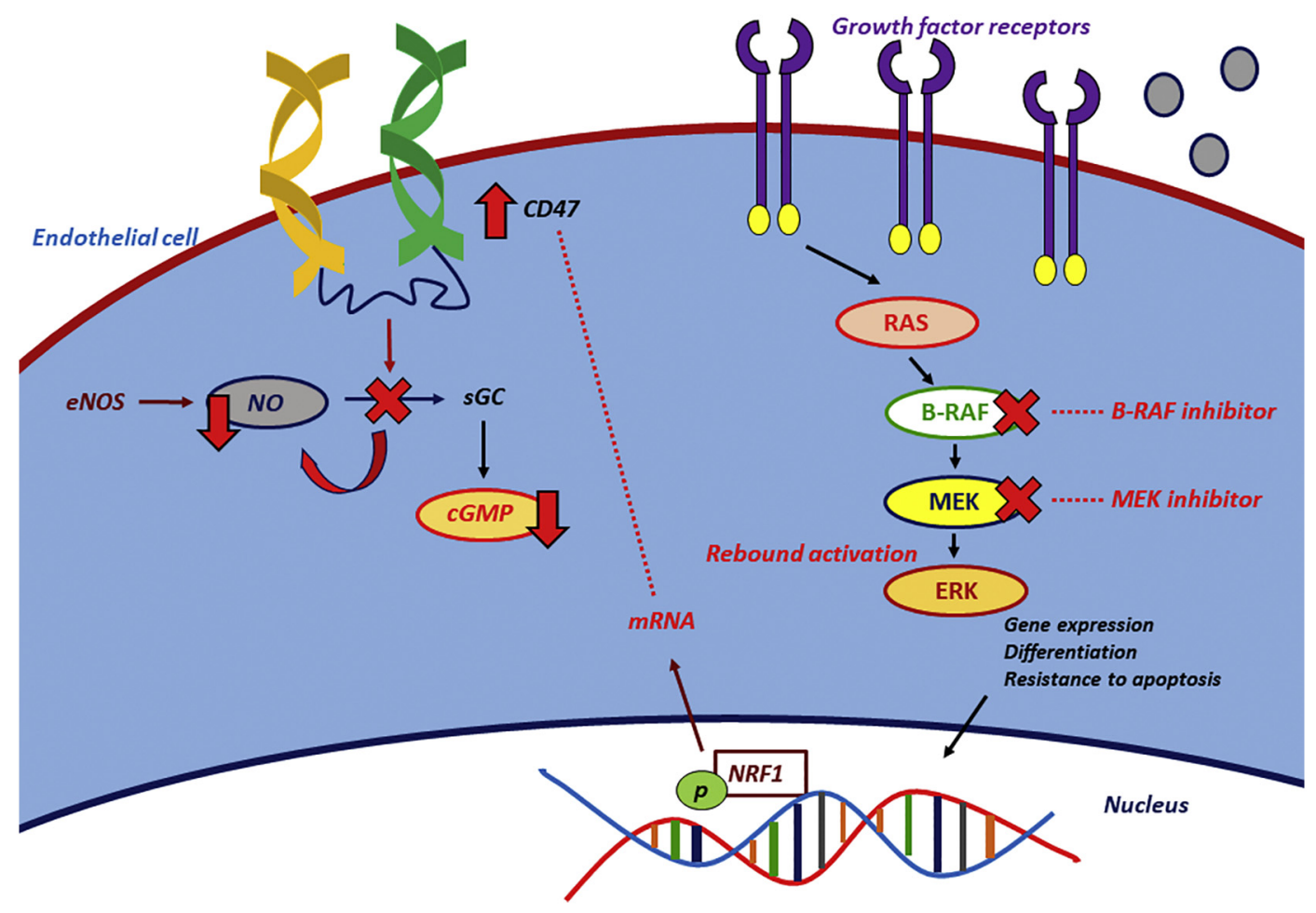

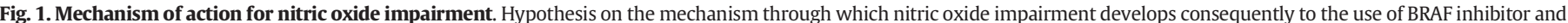

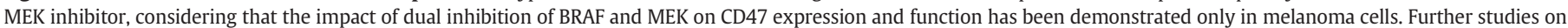

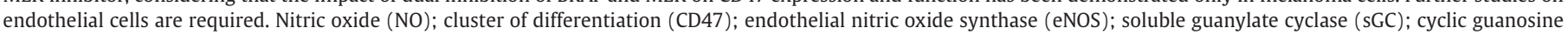
monophosphate (cGMP); nuclear respiratory factor 1 (NRF-1). 
activation of myosin light chain phosphatase, this leads to a rapid decrease in regulatory light chain-2 of myosin (MLC-2) phosphorylation and VSMC relaxation. Besides, NO initiates SGC in the endothelial cells determining a cGK-dependent, inhibitory phosphorylation of eNOS and termination of NO production, thus reinstating VSMC contractility. Another role carried out by NO is to maintain a balanced antithrombotic vascular system. The fluctations in NO levels can unbalance the slight equilibrium between pro- and anti-thrombotic states. It opposes platelets activation and aggregation. Even though it has to be specified that NO does not prevent platelet activation directly, but it increases the level of agonist required to initiate the process. (Isenberg, Roberts, \& Frazier, 2008)

So, ultimately the increase of CD47, induced by BRAF and MEK inhibition, cause a reduction in NO bioavailability. It has to be noted that this evidence came from a study on melanoma cells. At the current state of knowledge, it is only possible to hypothesize that these processes occur also in ECs and VSMCs, but further studies on these cells are necessary to confirm it. Hence, the reduction in NO bioavailability is followed by endothelial dysfunction and vasoconstriction. These could be the elements at the basis of hypertension caused by BRAF and MEK inhibition. (Isenberg et al., 2008) (Fig. 2).

Other molecules could be involved in those processes, which are responsible for the other cardiovascular adverse events. It has been demonstrated in Literature that estrogen (17ß-estradiol, E2) has a protective action on cardiovascular function. It interacts with three types of estrogen receptors (ERs), ER alpha (Esr1 or ER $\alpha$ ), ER beta (Esr2 or ER $\beta$ ) and the G-protein coupled estrogen receptor 1 (Gper1). These are all expressed in heart cells. The use of E2 prevents heart damage from ischemia/reperfusion $(\mathrm{I} / \mathrm{R})$ injury, this is given by the interaction with Gper1. In fact, knockout mice for Gper1, but not knockout mice for Esr1 and Esr2 showed in studies an ample infarcted area, even though they were treated with E2. This evidence suggests that Gper1 activation is necessary to protect the heart from infarct induced by I/R injury. The cardioprotective effect carried out through Gper1 is related to a decrease in mitochondrial permeability transition pore (mPTP) sensitivity to calcium $\left(\mathrm{Ca}^{2+}\right)$ overload. Besides, it has been highlighted that the modulation of some mediators is involved in the cardioprotective action triggered by the interaction of E2 with Gper1, which are an activation of Akt and ERK1/2 and the deactivation of GSK-3 3 . The E2-mediated Gper1 activation triggers two pathways. The first one is the MEK1/2-ERK1/2 pathway which leads to the phosphorylation of GSK-3 $\beta$, while the other one is the PI3K/Akt signaling pathway that does not cause the phosphorylation of GSK-3 $\beta$. Phosphorylated GSK$3 \beta$ inhibits the MPTP opening in response to various cardioprotective agents. Besides, evidence highlighted that the inhibition of PKC translocation suppresses the beneficial effect of E2 on heart functional recovery. So, this is proof that E2-Gper1 protective pathway in the mouse male heart involves also PKC. It could stimulate the MEK1/2-ERK1/2 pathway, which in turn induces GSK3- $\beta$ phosphorylation preventing opening of mPTP, or it could act straight to GSK3- $\beta$ to prevent mPTP opening. These evidences uphold once again the role that the RAF/ MEK/ERK signaling pathway has in cardioprotection, this is supported by several articles in Literature (Grohé, Kahlert, Löbbert, \& Vetter, 1998; Hausenloy \& Yellon, 2009; Kabir et al., 2015; Node et al., 1997)

Studies revealed how, in cardiomyocytes, the RAF/MEK/ERK pathway carries out in pressure-overloaded myocardium an important role for the development of hypertrophy and ribosomal biogenesis. It regulates hypertrophic gene induction, even though it could be not sufficient for cellular morphological changes necessary for cardiomyocyte hypertrophy. B-RAF particularly plays an important role in the regulation of cardiomyocyte hypertrophy. (Iijima et al., 2002; Kramann, Hasenfuß, \& Seidler, 2014).

Several studies demonstrated that ERK1/2 has cardioprotective effects. On one hand the mitochondrial ATP-sensitive $\mathrm{K}^{+}$channel (mitoK ${ }^{+}{ }_{\text {ATP }}$ ) activation, protects the mitochondrial function, on the other hand it limits the overproduction of cytotoxic reactive oxygen species (ROS) at the time of reperfusion in ischemic hearts. The opening of these channels stimulates ERK, this results in the suppression of gap junction permeability during an ischemic insult. This event leads to the suppression of propagation of myocyte injury limiting the infarcted area. So, ERK $1 / 2$ plays a role in the protection from oxidative stress, but also in adaptive hypertrophy and anti-apoptotic protection against

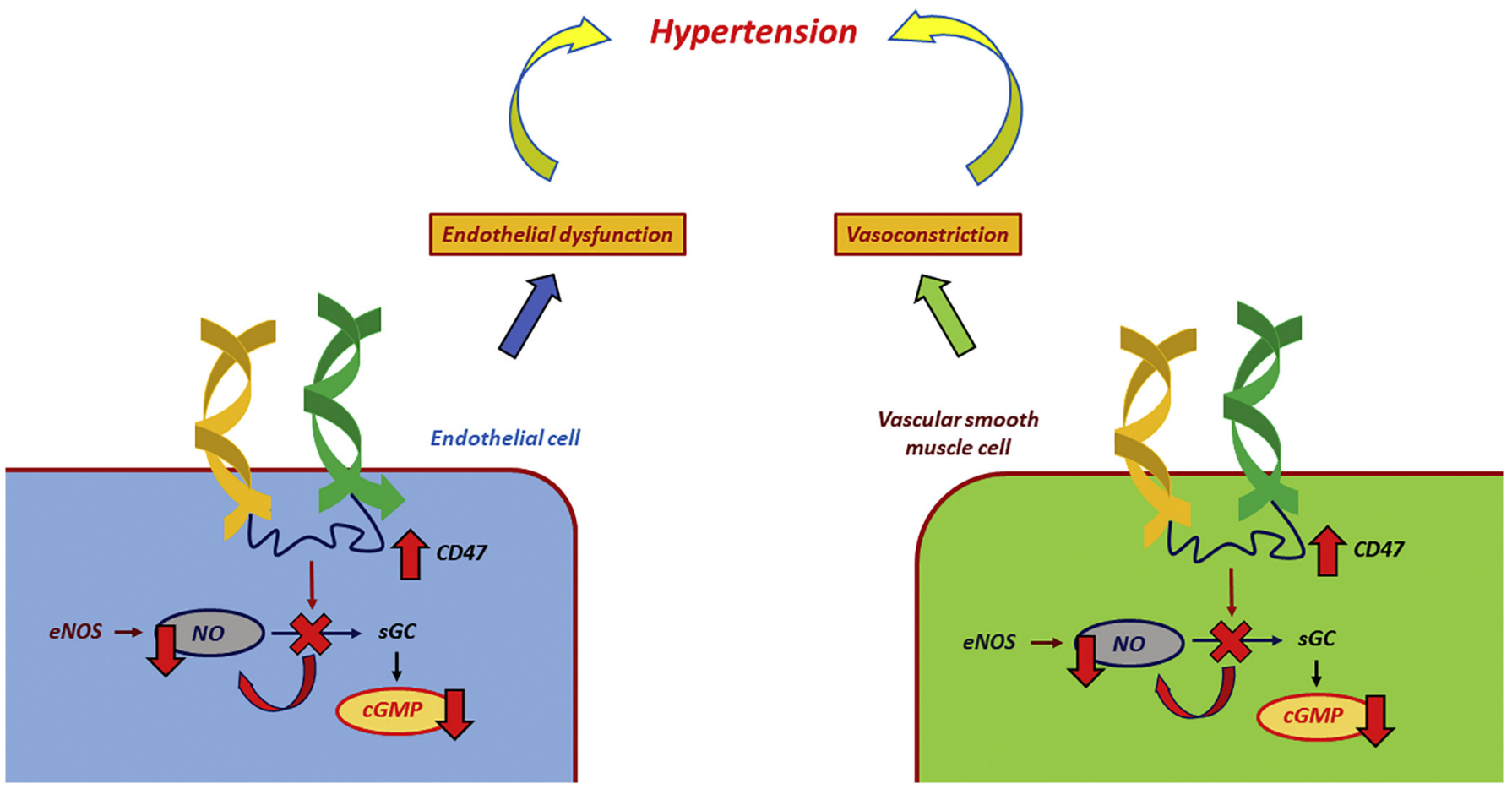

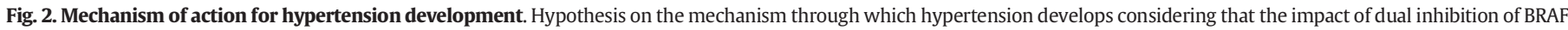

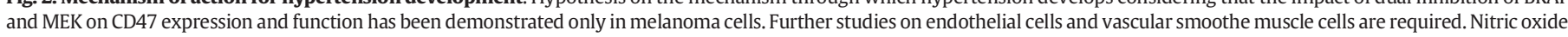
(NO); cluster of differentiation (CD47); endothelial nitric oxide synthase (eNOS); soluble guanylate cyclase (sGC); cyclic guanosine monophosphate (cGMP). 
cytotoxic insults. Knockout mice for ERK1/2 showed normal cardiac dimensions and function, but they are more prone to cardiomyocyte apoptosis and in developing larger infarcts if coronary arteries are blocked up. In fact, the reduction of ERK levels, causes a reduction in the production of $\mathrm{Bcl}-2$ and $\mathrm{Bcl}-\mathrm{xL}$ with consequential increased apoptosis. In normal conditions, if there are limitations in ERK function, the overexpression of MEK1 can balance this deficit. Hence the use of BRAF and MEK inhibitors make this compensatory mechanism vanish and cardioprotection can not be implemented (Rose et al., 2010) (Fig. 3). Ultimately, cardiomyocytes hypertrophy and cardiomyocytes damage or apoptosis are events that uphold the development of decreased ejection fraction, but it is important to consider that it could be a "two-hit" hypothesis that explains why in humans the cardiovascular toxicity from BRAF and MEK inhibitors is not so marked in some features. This means that it is the sum of two or more events causing damage, such as hypertension, ischemia or exposure to other toxic drugs that makes a cardiac event clinically revealed. Therefore, cardiomyocytes exposing to BRAF and MEK inhibitors could undergo apoptosis, but do not create a substantial heart damage. A second source of damage, such as hypertension that is directly linked to the combination therapy, then could give a greater damage determining, among others, the development of ejection fraction reduction. (Banks et al., 2017; Lips et al., 2004; Naitoh et al., 2006)

Besides, one of the key elements necessary for the formation of new arteries from already existing vessels or also in pathogenic events, such as restenosis, is the proliferation of smooth muscle cells. This proliferation is mainly linked to the activation of signaling pathway, which starts with the interaction of platelet derived growth factor (PDGF) and fibroblast growth factor (FGF) with their specific receptors. These molecules use the $\mathrm{RAS} / \mathrm{RAF} / \mathrm{MEK} / \mathrm{ERK}$ pathway to start smooth muscle cells proliferation.
Studies demonstrated that the inhibition of MEK1/2 in smooth muscle cells causes downregulation of de-differentiation, nuclear activation, DNAsynthesis, Rap2 mediated migration and increase in cell cycle inhibitory proteins. These events are necessary for arteriogenesis. (Kubin et al., 2017) So, the use of BRAFi and MEKi could create a microvascular rarefaction or damage that affects cardiac adaptation to afterload stress. This event if added to another cardiovascular event, e.g. hypertension, might support the long-term heart failure development.

\section{Cardiovascular toxicity by BRAF-inhibitor plus MEK-inhibitor}

\subsection{Dabrafenib plus Trametinib}

Dabrafenib and Trametinib have been studied in two phase 3 studies, COMBI-d and COMBI-v. In the first one, Long et al. screened 947 patients who did not take a previous therapy and had a diagnosis of unresectable stage IIIC or stage IV melanoma with a positive mutational status for BRAF V600 (V600E or V600K). 423 of these patients were randomized to receive Dabrafenib ( $150 \mathrm{mg}$ orally twice daily) plus Trametinib (2 mg orally once daily) or Dabrafenib plus placebo. All grades hypertension was reported in the Dabrafenib-Trametinib group in $22 \%$ of the patients and $4 \%$ of these patients had grade 3 hypertension. All grade hypertension was higher than the Dabrafenib group. They also reported in this work peripheral oedema and decreased ejection fraction (Table 1). 4 patients who received Dabrafenib-Trametinib died for adverse events, even though investigators considered them unrelated to the treatment, 3 of these patients died for cerebral hemorrhage. (Long et al., 2014) These data were updated by the authors in a work published in 2015. They reported any grade decreased ejection fraction in 9 patients (4\%), of which 6 (3\%) had grade 2 and 3 patients (1\%)

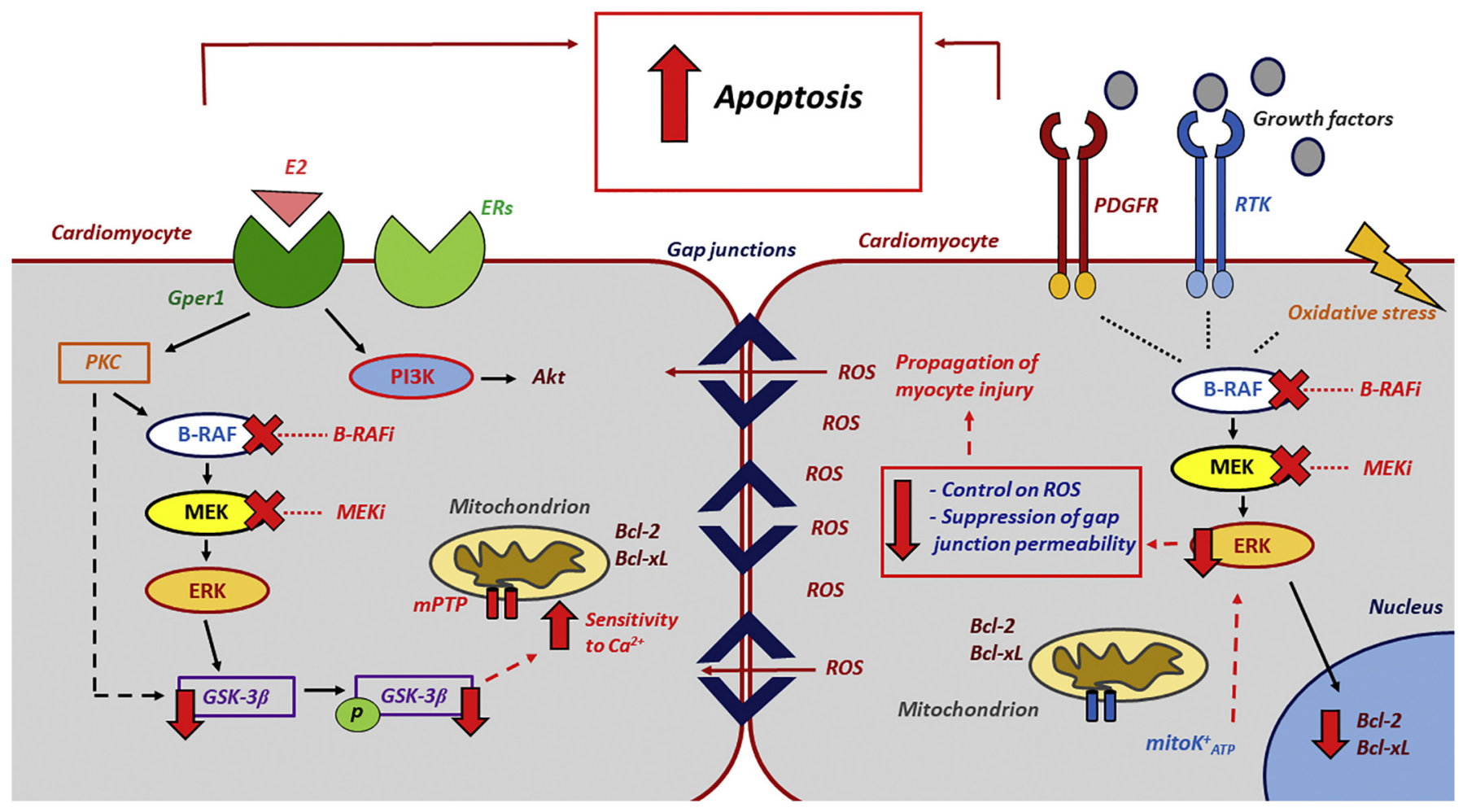

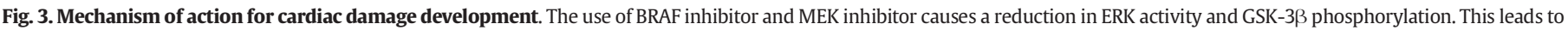

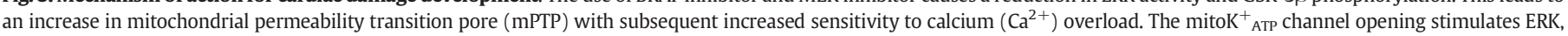

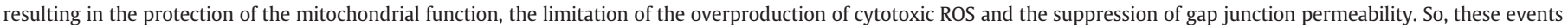

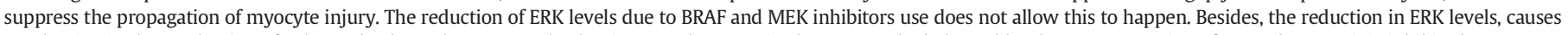

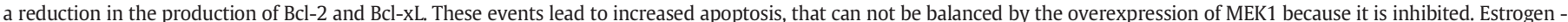

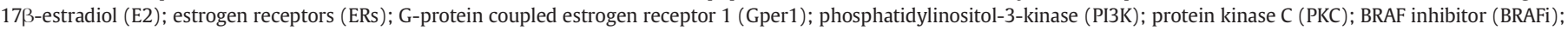

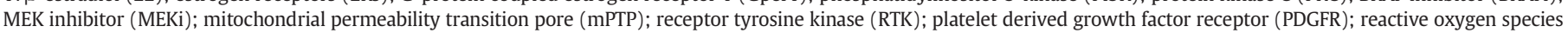
(ROS); mitochondrial ATP-sensitive $\mathrm{K}+$ channel ( mitoK $^{+}{ }_{\text {ATP }}$ ). 
had grade 3.3 patients who had this adverse event (1\%) discontinued treatment. All grades fatigue was registered in 56 patients (27\%), of which $20(10 \%)$ had grade 2 and $4(2 \%)$ had grade 3.22 patients had all grades peripheral oedema (11\%), 3 (1\%) presented grade 2 , while 2 (1\%) had grade 3. (Long et al., 2015)
In the phase 3 trial COMBI-v, Robert et al. screened 1645 patients. 704 patients affected by metastatic melanoma BRAF V600 mutated (V600E - V600 K) were randomized to receive Dabrafenib (150 mg twice daily) and Trametinib (2 $\mathrm{mg}$ once daily) or Vemurafenib (960 mg twice daily) orally as the first-line therapy. In this work the

Table 1

Percentages of each cardiovascular side effect linked to BRAF-inhibitor plus MEK-inhibitor from phase 2 and 3 clinical trials.

\begin{tabular}{|c|c|c|c|c|c|c|c|c|c|c|}
\hline Study (Reference) & $\begin{array}{l}\text { Treatment } \\
\text { arms }\end{array}$ & $\begin{array}{l}\text { Adverse } \\
\text { event grade } \\
\% \text { (number of } \\
\text { patients) }\end{array}$ & $\begin{array}{l}\text { Hypertension } \\
\%(\mathrm{n})\end{array}$ & $\begin{array}{l}\text { QT-interval } \\
\text { prolongation }\end{array}$ & $\begin{array}{l}\text { Decreased } \\
\text { ejection } \\
\text { fraction }\end{array}$ & $\begin{array}{l}\text { Peripheral } \\
\text { oedema }\end{array}$ & Asthenia & Dyspnoea & $\begin{array}{l}\text { Asymptomatic } \\
\text { increased creatine } \\
\text { kinase }\end{array}$ & Fatigue \\
\hline \multirow[t]{4}{*}{$\begin{array}{l}\text { COMBI-d - phase } 3 \\
\text { (Long et al., 2014) }\end{array}$} & \multirow[t]{2}{*}{$\begin{array}{l}\text { Dabrafenib } \\
+ \text { Trametinib }\end{array}$} & All grade & $22(46)$ & Not reported & $4(9)$ & $14(30)$ & $10(20)$ & $\begin{array}{l}\text { Not } \\
\text { reported }\end{array}$ & Not reported & $\begin{array}{l}35 \\
(74)\end{array}$ \\
\hline & & Grade $\geq 3$ & $4(8)$ & Not reported & $<1(1)$ & $<1(1)$ & $<1(1)$ & $\begin{array}{l}\text { Not } \\
\text { reported }\end{array}$ & Not reported & $2(4)$ \\
\hline & \multirow[t]{2}{*}{ Dabrafenib } & All grade & $14(29)$ & Not reported & $2(5)$ & $5(10)$ & $13(27)$ & $\begin{array}{l}\text { Not } \\
\text { reported }\end{array}$ & Not reported & $\begin{array}{l}35 \\
(74)\end{array}$ \\
\hline & & Grade $\geq 3$ & $5(10)$ & Not reported & $<1(1)$ & $<1(1)$ & $<1(1)$ & $\begin{array}{l}\text { Not } \\
\text { reported }\end{array}$ & Not reported & $1(2)$ \\
\hline \multirow[t]{4}{*}{$\begin{array}{l}\text { COMBI-d (update) - phase } 3 \\
\quad \text { (Long et al., 2015) }\end{array}$} & \multirow[t]{2}{*}{$\begin{array}{l}\text { Dabrafenib } \\
+ \text { Trametinib }\end{array}$} & All grade & Not reported & Not reported & $4(9)$ & $11(22)$ & $\begin{array}{l}\text { Not } \\
\text { reported }\end{array}$ & $\begin{array}{l}\text { Not } \\
\text { reported }\end{array}$ & Not reported & $\begin{array}{l}27 \\
(56)\end{array}$ \\
\hline & & Grade $\geq 3$ & Not reported & Not reported & $1(3)$ & $1(2)$ & $\begin{array}{l}\text { Not } \\
\text { reported }\end{array}$ & $\begin{array}{l}\text { Not } \\
\text { reported }\end{array}$ & Not reported & $2(4)$ \\
\hline & \multirow[t]{2}{*}{ Dabrafenib } & All grade & Not reported & Not reported & $3(7)$ & $2(4)$ & $\begin{array}{l}\text { Not } \\
\text { reported }\end{array}$ & $\begin{array}{l}\text { Not } \\
\text { reported }\end{array}$ & Not reported & $\begin{array}{l}28 \\
(59)\end{array}$ \\
\hline & & Grade $\geq 3$ & Not reported & Not reported & $2(4)$ & $0(0)$ & $\begin{array}{l}\text { Not } \\
\text { reported }\end{array}$ & $\begin{array}{l}\text { Not } \\
\text { reported }\end{array}$ & Not reported & $1(2)$ \\
\hline \multirow[t]{4}{*}{$\begin{array}{l}\text { COMBI-v - phase } 3 \\
\text { (Robert et al., 2015) }\end{array}$} & \multirow[t]{2}{*}{$\begin{array}{l}\text { Dabrafenib } \\
+ \text { Trametinib }\end{array}$} & All grade & $26(92)$ & Not reported & $8(29)$ & $12(42)$ & $16(55)$ & $\begin{array}{l}\text { Not } \\
\text { reported }\end{array}$ & Not reported & $\begin{array}{l}29 \\
(101)\end{array}$ \\
\hline & & Grade $\geq 3$ & $14(48)$ & Not reported & $4(13)$ & $<1(1)$ & $1(4)$ & $\begin{array}{l}\text { Not } \\
\text { reported }\end{array}$ & Not reported & $1(4)$ \\
\hline & \multirow[t]{2}{*}{ Vemurafenib } & All grade & $24(84)$ & Not reported & $0(0)$ & $10(35)$ & $16(57)$ & $\begin{array}{l}\text { Not } \\
\text { reported }\end{array}$ & Not reported & $\begin{array}{l}33 \\
(115)\end{array}$ \\
\hline & & Grade $\geq 3$ & $9(32)$ & Not reported & $0(0)$ & $<1(1)$ & $1(4)$ & $\begin{array}{l}\text { Not } \\
\text { reported }\end{array}$ & Not reported & $2(6)$ \\
\hline \multirow[t]{2}{*}{$\begin{array}{l}\text { NCT01336634 - phase } 2 \\
\quad \text { (Planchard et al., 2016) }\end{array}$} & \multirow[t]{2}{*}{$\begin{array}{l}\text { Dabrafenib } \\
+ \text { Trametinib }\end{array}$} & All grade & $6(3)$ & Not reported & $\begin{array}{l}\text { Not } \\
\text { reported }\end{array}$ & $23(13)$ & $32(18)$ & $18(10)$ & Not reported & $\begin{array}{l}18 \\
(10)\end{array}$ \\
\hline & & Grade $\geq 3$ & $4(2)$ & Not reported & $\begin{array}{l}\text { Not } \\
\text { reported }\end{array}$ & $0(0)$ & $4(2)$ & $4(2)$ & Not reported & $2(1)$ \\
\hline \multirow[t]{4}{*}{$\begin{array}{l}\text { COMBI-AD - phase } 3 \\
\text { (Long et al., 2017) }\end{array}$} & \multirow[t]{2}{*}{$\begin{array}{l}\text { Dabrafenib } \\
+ \text { Trametinib }\end{array}$} & All grade & $11(49)$ & Not reported & $\begin{array}{l}\text { Not } \\
\text { reported }\end{array}$ & $13(58)$ & $13(58)$ & $\begin{array}{l}\text { Not } \\
\text { reported }\end{array}$ & Not reported & $\begin{array}{l}47 \\
(204)\end{array}$ \\
\hline & & Grade $\geq 3$ & $6(25)$ & Not reported & $\begin{array}{l}\text { Not } \\
\text { reported }\end{array}$ & $<1(1)$ & $<1(2)$ & $\begin{array}{l}\text { Not } \\
\text { reported }\end{array}$ & Not reported & $4(19)$ \\
\hline & \multirow[t]{2}{*}{ Placebo } & All grade & $8(35)$ & Not reported & $\begin{array}{l}\text { Not } \\
\text { reported }\end{array}$ & $4(19)$ & $10(42)$ & $\begin{array}{l}\text { Not } \\
\text { reported }\end{array}$ & Not reported & $\begin{array}{l}28 \\
(122)\end{array}$ \\
\hline & & Grade $\geq 3$ & $2(8)$ & Not reported & $\begin{array}{l}\text { Not } \\
\text { reported }\end{array}$ & $0(0)$ & $<1(1)$ & $\begin{array}{l}\text { Not } \\
\text { reported }\end{array}$ & Not reported & $<1(1)$ \\
\hline \multirow[t]{4}{*}{$\begin{array}{l}\text { coBRIM - phase } 3 \text { (Larkin } \\
\text { et al., 2014) }\end{array}$} & \multirow[t]{2}{*}{$\begin{array}{l}\text { Vemurafenib } \\
+ \text { Cobimetinib }\end{array}$} & All grade & Not reported & $4(9)$ & $8(19)$ & $\begin{array}{l}\text { Not } \\
\text { reported }\end{array}$ & $\begin{array}{l}\text { Not } \\
\text { reported }\end{array}$ & $\begin{array}{l}\text { Not } \\
\text { reported }\end{array}$ & $31(76)$ & $\begin{array}{l}32 \\
(81)\end{array}$ \\
\hline & & Grade $\geq 3$ & Not reported & $<1(1)$ & $1(3)$ & $\begin{array}{l}\text { Not } \\
\text { reported }\end{array}$ & $\begin{array}{l}\text { Not } \\
\text { reported }\end{array}$ & $\begin{array}{l}\text { Not } \\
\text { reported }\end{array}$ & $11(26)$ & $4(9)$ \\
\hline & \multirow[t]{2}{*}{ Vemurafenib } & All grade & Not reported & $5(13)$ & $3(7)$ & $\begin{array}{l}\text { Not } \\
\text { reported }\end{array}$ & $\begin{array}{l}\text { Not } \\
\text { reported }\end{array}$ & $\begin{array}{l}\text { Not } \\
\text { reported }\end{array}$ & $4(7)$ & $\begin{array}{l}31 \\
(73)\end{array}$ \\
\hline & & Grade $\geq 3$ & Not reported & $1(3)$ & $1(3)$ & $\begin{array}{l}\text { Not } \\
\text { reported }\end{array}$ & $\begin{array}{l}\text { Not } \\
\text { reported }\end{array}$ & $\begin{array}{l}\text { Not } \\
\text { reported }\end{array}$ & $0(0)$ & $3(7)$ \\
\hline \multirow[t]{4}{*}{$\begin{array}{l}\text { coBRIM (update) - phase } 3 \\
\text { (Ascierto et al., 2016) }\end{array}$} & \multirow[t]{2}{*}{$\begin{array}{l}\text { Vemurafenib } \\
\text { +Cobimetinib }\end{array}$} & All grade & $15.8(39)$ & $5(11)$ & $12(29)$ & $13.8(34)$ & $19(47)$ & $7.7(19)$ & $35(87)$ & $\begin{array}{l}37 \\
(91)\end{array}$ \\
\hline & & Grade $\geq 3$ & $6(15)$ & $1(3)$ & $2(5)$ & $0(0)$ & $2(5)$ & $0(0)$ & $12(30)$ & $5(11)$ \\
\hline & \multirow[t]{2}{*}{ Vemurafenib } & All grade & $8(20)$ & $5(13)$ & $5(13)$ & $11(28)$ & $17(43)$ & $7(18)$ & $3(7)$ & $\begin{array}{l}33 \\
(82)\end{array}$ \\
\hline & & Grade $\geq 3$ & $3(7)$ & $1(3)$ & $1(3)$ & $<1(1)$ & $1(3)$ & $1(2)$ & $<1(1)$ & $3(7)$ \\
\hline \multirow[t]{6}{*}{$\begin{array}{l}\text { COLUMBUS - phase } 3 \\
\text { (Dummer et al., 2018) }\end{array}$} & \multirow[t]{2}{*}{$\begin{array}{l}\text { Encorafenib } \\
+ \text { Binimetinib }\end{array}$} & All grade & $11(21)$ & $0(0)$ & $6(11)$ & $2(3)$ & $19(35)$ & $7(14)$ & $23(44)$ & $\begin{array}{l}29 \\
(55)\end{array}$ \\
\hline & & Grade $\geq 3$ & $6(11)$ & $0(0)$ & $1(2)$ & $0(0)$ & $2(3)$ & $1(1)$ & $7(13)$ & $2(4)$ \\
\hline & \multirow[t]{2}{*}{ Encorafenib } & All grade & $6(11)$ & $4(7)$ & $2(4)$ & $2(3)$ & $19(37)$ & $5(9)$ & $1(2)$ & $\begin{array}{l}25 \\
(48)\end{array}$ \\
\hline & & Grade $\geq 3$ & $3(6)$ & $1(1)$ & $1(2)$ & $0(0)$ & $2(5)$ & $1(1)$ & $0(0)$ & $1(1)$ \\
\hline & \multirow[t]{2}{*}{ Vemurafenib } & All grade & $11(21)$ & $3(6)$ & $1(1)$ & $4(7)$ & $18(34)$ & $9(16)$ & $2(4)$ & $\begin{array}{l}31 \\
(57)\end{array}$ \\
\hline & & Grade $\geq 3$ & $3(6)$ & $0(0)$ & $0(0)$ & $1(1)$ & $4(8)$ & $2(3)$ & $0(0)$ & $2(4)$ \\
\hline
\end{tabular}

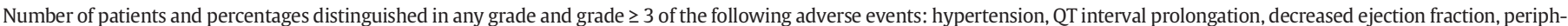
eral oedema, asthenia, dyspnoea, asymptomatic increased creatine kinase, fatigue. Besides data are distinguished for clinical trial and treatment arms.

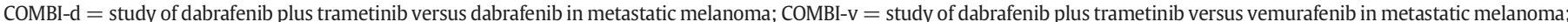

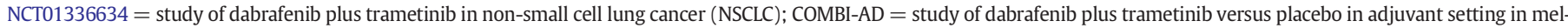

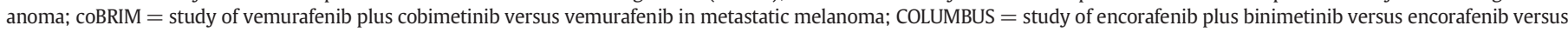
vemurafenib in metastatic melanoma. 
authors registered decreased ejection fraction in the DabrafenibTrametinib group, that was not in the Vemurafenib group. Little difference between the two groups was reported for fatigue and hypertension. Substantially the same data were reported in the two groups about asthenia, while a sharp difference was reported for any grade peripheral oedema between the two groups (see Table 1). (Robert et al., 2015)

The combination therapy Dabrafenib plus Trametinib has been also studied in a phase 2 trial (NCT01336634) in 57 patients affected by non-small cell lung cancer, who were previously treated with systemic chemotherapy for metastatic disease. These patients presented the mutation BRAF V600E. The authors reported hypertension, peripheral oedema, asthenia, dyspnoea and fatigue (see Table 1 ). Besides, hypotension was present in 7 patients (12\%), all of them grade 1-2 and chest pain in 6 patients (11\%), all of them grade 1-2. (Planchard et al., 2016)

These data were updated in a work published in 2017 showing different percentages in cardiovascular toxicity. This was probably due to the small number of patients treated. They were 36 patients. This work registered: fatigue in $36 \%$ of patients (grade 1-2), peripheral oedema in $36 \%$ (grade $1-2$ ), dyspnoea grade $1-2$ in $11 \%$ and grade 3 in $6 \%$, hypotension grade $1-2$ in $11 \%$ and grade 3 in $6 \%$, hypertension grade 3 in $11 \%$, asthenia grade $1-2$ in $8 \%$ and grade 3 in $3 \%$, ejection fraction decrease grade $1-2$ in $3 \%$ and grade 3 in $6 \%$, pulmonary embolism grade $1-2$ in $3 \%$ and grade 3 in $6 \%$, blood creatine phosphokinase increase grade 3 in 3\%, cardiac arrest grade 5 in 3\%. (Planchard et al., 2017)

These two drugs have been also studied in the adjuvant setting in melanoma. These data were recently published. These are data from a double-blind, placebo-controlled, phase 3 trial, the COMBI-AD trial. 870 patients with completely resected, stage III melanoma with BRAF V600E or V600 K mutations were randomly assigned to receive oral Dabrafenib plus Trametinib or placebo tablets for 12 months. Authors reported hypertension, fatigue, asthenia, peripheral oedema. No data were reported along the full-text about decreased ejection fraction, dyspnoea, QT-interval prolongation and asymptomatic increased creatine kinase (see Table 1). (Long et al., 2017)

Very different data about cardiovascular toxicity emerged from a phase 2 trial in which this combination therapy was studied in the neoadjuvant setting: fatigue grade $1-2$ in $85 \%$, hypertension grade $1-2$ in $54 \%$, grade 3 atrial fibrillation in $8 \%$, grade 3 syncope in $8 \%$. This was probably due to the small number of patients, because only 13 patients received the treatment Dabrafenib and Trametinib. (Amaria et al., 2018)

\subsection{Vemurafenib plus Cobimetinib}

The combination therapy Vemurafenib plus Cobimetinib has been studied in the coBRIM trial (NCT01689519). 1049 patients who had a diagnosis of metastatic melanoma in presence of the mutation BRAF V600 were screened in the study, but only 495 were randomized to receive Vemurafenib and Cobimetinib or Vemurafenib and placebo. Of the 254 patients treated with the combination therapy, 6 patients (2\%) had grade 1 QT-interval prolongation, $2(1 \%)$ had grade 2,1 patient $(<1 \%)$ had grade 3 , no grade 4 QT-interval prolongation were reported. These data were roughly almost the same to the QT-interval prolongation data registered for the therapy Vemurafenib and placebo. However, different results were recorded for decreased ejection fraction. These percentages were higher than the Vemurafenib-placebo arm. Asymptomatic increased creatine kinase level, in patients who took the combination therapy was more pronounced than the Vemurafenib-placebo arm (see Table 1) (Larkin et al., 2014). These data were updated in a work by Ascierto et al. They registered hypertension, peripheral oedema, asthenia, dyspnoea, decreased left ventricular ejection fraction, QT prolongation, fatigue and blood creatine phosphokinase level increase (see Table 1). Some symptoms were present in low percentage, such as chest pain ( 5 patients - $2 \%$ all grades, of which 4 patients had grade 1 ) and hypotension ( 7 patients $-2.8 \%$ all grades, of which 3 patients grade 1 and 3 patients grade 2 ). Finally, 32 patients presented all grades cardiac disorders (13\%) (17 patients grade $1-6.9 \%, 8$ patients grade $2-3.2 \%$, 6 patients grade $3-2.4 \%$, one patient grade $5-0.4 \%) .5$ patients $(2 \%)$ of 247 patients treated in the combination therapy arm died during the study, one of them had cardiac arrest. (Ascierto et al., 2016)

\subsection{Encorafenib plus Binimetinib}

The other combination therapy studied was Encorafenib and Binimetinib, that has been evaluated in the COLUMBUS trial. Researchers evaluated 577 patients affected by locally advanced unresectable or metastatic melanoma presenting BRAF V600 mutation. They compared 192 patients receiving Encorafenib 450 mg once a day plus Binimetinib $45 \mathrm{mg}$ twice a day (COMBO450) with 194 patients assuming Encorafenib 300 mg once a day (ENCO300) and with 191 patients receiving Vemurafenib $960 \mathrm{mg}$ twice a day. In the combination treatment arm, it was registered any grade fatigue in $29 \%$, grade $\geq 3$ was present in $2 \% .23 \%$ of patients had any grade blood creatine phosphokinase increase, $7 \%$ was grade $\geq 3$. Any grade hypertension was highlighted in $11 \%$ of patients, of which $6 \%$ was grade $\geq 3$. Left ventricular dysfunction of any grade was registered in $8 \%$ of patients, of which $2 \%$ had grade $\geq 3$. Finally, grade $1-2$ asthenia was present in 32 patients (17\%), two patients had grade 3 asthenia (1\%) and one patient had grade 4 asthenia (1\%). (Dummer et al., 2018)

\section{Perspectives of combination therapy}

In view of these significant results obtained from the combination therapy BRAFi plus MEKi and considering the important effect that this has on the immune system, many other studies were designed and started in recent years. Thus, new combinations are under investigation in ongoing trials, including BRAFi and MEKi. These two drugs are tested in combination with immunotherapy (anti-PD(Programmed Cell Death Protein)-1/Programmed Cell Death Protein-Ligand 1 (PD-L1) agents) or Bcl-2 inhibitor (Navitoclax) or Hsp90 Inhibitor (Onalespib) or Melanoma Helper Peptide Vaccine (6MHP) or anti-EGFR (Panitumumab) or anti-VEGF (Bevacizumab). The trials still recruiting are: a. Dabrafenib, Trametinib, and Navitoclax in treating patients with BRAF mutant melanoma or solid tumors that are metastatic or cannot be removed by surgery (NCT01989585); b. Hsp90 Inhibitor AT13387 (Onalespib), Dabrafenib, and Trametinib in treating patients with recurrent melanoma or solid tumors that are metastatic or cannot be removed by surgery (NCT02097225); c. a clinical trial to evaluate a Melanoma Helper Peptide Vaccine (6MHP) plus Dabrafenib and Trametinib (NCT02382549); d. study of the anti-PD-1 antibody Nivolumab in combination with Dabrafenib and/or Trametinib in patients with BRAF or NRAS-mutated metastatic melanoma (NCT02910700); e. a study of the safety and efficacy of Pembrolizumab (MK-3475) in combination with Trametinib and Dabrafenib in participants with advanced melanoma (MK-3475-022/KEYNOTE-022) (NCT02130466); f. a study of Atezolizumab plus Cobimetinib and Vemurafenib versus placebo plus Cobimetinib and Vemurafenib in previously untreated BRAFV600 mutation-positive participants with metastatic or unresectable locally advanced melanoma (NCT02908672); g. neoadjuvant Dabrafenib, Trametinib and/or Pembrolizumab in BRAF mutant resectable stage III melanoma (NCT02858921); h. open label, multicenter study of Encorafenib + Binimetinib + PD-1 antibody Pembrolizumab (NCT02902042). A study active, but not recruiting, is: BRAF/MEK/EGFR inhibitor combination study in colorectal cancer (CRC) (NCT01750918). Another study evaluating the use of Ipililumab (anti-CTLA-4) in combination with Dabrafenib and Trametinib is already completed: study of Dabrafenib $+/-$ Trametinib in combination with Ipilimumab for $\mathrm{V} 600 \mathrm{E} / \mathrm{K}$ mutation positive metastatic or unresectable melanoma (NCT01767454), of which data are awaited, as well as, phase 1 safety and tolerability of MEDI4736 in combination with Dabrafenib and Trametinib or with Trametinib alone (NCT02027961). The trial of Vemurafenib/Cobimetinib with or without 
Bevacizumab in patients with stage IV BRAFV600 mutant melanoma is completed and it has results (NCT01495988). (Ascierto, Marincola, \& Atkins, 2015; Queirolo, Picasso, \& Spagnolo, 2015; http://ascopubs.org/ doi/abs/10.1200/jco.2015.33.15_suppl.3003; https://clinicaltrials.gov).

\section{Discussion and conclusions}

In last years there has been a growing use of combination therapies in Oncology and many others are still under investigation. The use of drugs in combination led to a significant improvement of tumor responses but paying the price of an increased toxicity, included the cardiovascular toxicity. Until now, many drugs used in monotherapy caused a restrained cardiovascular toxicity but using drugs in combination might bring much more cardiovascular toxicity, determining new therapeutic challenges for Oncologists and Cardiologists. In fact, the drugs used to date to treat certain cardiovascular symptoms may not be adequate to treat the same symptoms that represent a treatmentrelated adverse event.

We aimed in this work to highlight the mechanisms that underlie these adverse events and the percentages emerged from phase 2 and 3 clinical trials data about each cardiovascular adverse event (Table 1). Taking a look at these data, it apparently seems that the combination of BRAF inhibitor and MEK inhibitor gives an increased cardiovascular toxicity compared to the monotherapy with BRAF inhibitor, especially as regards hypertension and decreased ejection fraction, even though it would be possible to obtain definite conclusions only through a rigorous statistical analysis of the data. Besides, to our knowledge until now there is not a defined protocol to monitor patients undergoing treatment with BRAF inhibitor and MEK inhibitor, therefore cardiovascular monitoring is discretional. Hence, prospective studies are necessary to define a protocol for these patients and to find some markers that could highlight a subclinical damage, such as global longitudinal strain at echocardiography or serum biomarkers such as troponins, brain natriuretic peptides or microRNAs.

In this work we described various processes and we described the mechanisms involved in the development of cardiovascular adverse events linked to the use of BRAF inhibitor and MEK inhibitor. It is important to highlight that some of them, such as the impact of dual inhibition of BRAF and MEK on CD47 expression and function, the rebound activation of ERK consequential to BRAF and MEK inhibition and the paradoxical hyperactivation of MEK caused by BRAF inhibition, have been only studied in melanoma cells. To best of our knowledge, there are no studies that evaluate these processes in endothelial cells, smooth muscle cells or cardiomyocytes. Hence, the pathophysiological mechanisms of cardiovascular toxicity described in this paper were gathered on the basis of indirect evidence. For this reason, pre-clinical studies are necessary to reinforce these hypotheses to achieve a better knowledge about the cardiovascular toxicity due to the use of BRAFi and MEKi.

There is still much to know and to study about this topic. However, this work represents the first piece in the study of this field in order to offer to researchers the basic tools. A careful study of this topic and development of a defined protocol would allow to treat in the best possible way these side effects and maintain anti-tumor treatment for as long as it is possible, so that Oncologist should not suspend it due to a side effect.

\section{Conflicts of interest statement}

The authors declare that there are no conflicts of interests.

\section{References}

Alsina, J., Gorsk, D. H., Germino, F. J., Shih, W., Lu, S. E., Zhang, Z. G., et al. (2003). Detection of mutations in the mitogen-activated protein kinase pathway in human melanoma. Clinical Cancer Research 9(17), 6419-6425.

Amaria, R. N., Prieto, P. A., Tetzlaff, M. T., Reuben, A., Andrews, M. C., Ross, M. I., et al. (2018). Neoadjuvant plus adjuvant dabrafenib and trametinib versus standard of care in patients with high-risk, surgically resectable melanoma: A single-centre, open-label, randomised, phase 2 trial. The Lancet Oncology 19(2), 181-193.

Ascierto, P. A., Marincola, F. M., \& Atkins, M. B. (2015). What's new in melanoma? Combination! Journal of Translational Medicine 13, 213.

Ascierto, P. A., McArthur, G. A., Dréno, B., Atkinson, V., Liszkay, G., Di Giacomo, A. M., et al (2016). Cobimetinib combined with vemurafenib in advanced BRAF(V600)-mutant melanoma (coBRIM): Updated efficacy results from a randomised, double-blind, phase 3 trial. The Lancet Oncology 17(9), 1248-1260.

Banks, M., Crowell, K., Proctor, A., \& Jensen, B. C. (2017). Cardiovascular effects of the MEK inhibitor, Trametinib: A case report, literature review, and consideration of mechanism. Cardiovascular Toxicology 17(4), 487-493.

Bronte, E., Bronte, G., Novo, G., Bronte, F., Bavetta, M. G., Lo Re, G., et al. (2015). What links BRAF to the heart function? New insights from the cardiotoxicity of BRAF inhibitors in cancer treatment. Oncotarget 6(34), 35589-35601.

Bronte, G., Bronte, E., Novo, G., Pernice, G., Lo Vullo, F., Musso, E., et al. (2015). Conquests and perspectives of cardio-oncology in the field of tumor angiogenesis-targeting tyrosine kinase inhibitor-based therapy. Expert Opinion on Drug Safety 14(2), 253-267.

Cardinale, D., Colombo, A., Bacchiani, G., Tedeschi, I., Meroni, C. A., Veglia, F., et al. (2015) Early detection of anthracycline cardiotoxicity and improvement with heart failure therapy. Circulation 131(22), 1981-1988.

Cartlidge, R. A., Thomas, G. R., Cagnol, S., Jong, K. A., Molton, S. A., Finch, A. J., et al. (2008) Oncogenic BRAF(V600E) inhibits BIM expression to promote melanoma cell survival. Pigment Cell \& Melanoma Research 21(5), 534-544.

Collisson, E. A., De, A., Suzuki, H., Gambhir, S. S., \& Kolodney, M. S. (2003). Treatment of metastatic melanoma with an orally available inhibitor of the Ras-Raf-MAPK cascade. Cancer Research 63(18), 5669-5673.

Davies, H., Bignell, G. R., Cox, C., Stephens, P., Edkins, S., Clegg, S., et al. (2002). Mutations of the BRAF gene in human cancer. Nature 417(6892), 949-954.

Di Lisi, D., Madonna, R., Zito, C., Bronte, E., Badalamenti, G., Parrella, P., et al. (2017). Anticancer therapy-induced vascular toxicity: VEGF inhibition and beyond. International Journal of Cardiology 227, 11-17.

Dossett, L. A., Kudchadkar, R. R., \& Zager, J. S. (2015). BRAF and MEK inhibition in melanoma. Expert Opinion on Drug Safety 14(4), 559-570.

Dummer, R., Ascierto, P. A., Gogas, H. J., Arance, A., Mandala, M., Liszkay, G., et al. (2018). Encorafenib plus binimetinib versus vemurafenib or encorafenib in patients with BRAF-mutant melanoma (COLUMBUS): A multicentre, open-label, randomised phase 3 trial. The Lancet Oncology 19(5), 603-615.

Flaherty, K. T., Infante, J. R., Daud, A., Gonzalez, R., Kefford, R. F., Sosman, J., et al. (2012). Combined BRAF and MEK inhibition in melanoma with BRAF V600 mutations. The New England Journal of Medicine 367(18), 1694-1703.

Flaherty, K. T., Robert, C., Hersey, P., Nathan, P., Garbe, C., Milhem, M., et al. (2012). Improved survival with MEK inhibition in BRAF-mutated melanoma. The New England Journal of Medicine 367(2), 107-114.

Grohé, C., Kahlert, S., Löbbert, K., \& Vetter, H. (1998). Expression of oestrogen receptor alpha and beta in rat heart: Role of local oestrogen synthesis. The Journal of Endocrinology 156(2), R1-R7.

Hauschild, A., Grob, J. J., Demidov, L. V., Jouary, T., Gutzmer, R., Millward, M., et al. (2012) Dabrafenib in BRAF-mutated metastatic melanoma: A multicentre, open-label, phase 3 randomised controlled trial. Lancet 380(9839), 358-365.

Hausenloy, D. J., \& Yellon, D. M. (2009). Cardioprotective growth factors. Cardiovascular Research 83(2), 179-194.

http://ascopubs.org/doi/abs/10.1200/jco.2015.33.15_suppl.3003.

https://clinicaltrials.gov.

Iijima, Y., Laser, M., Shiraishi, H., Willey, C. D., Sundaravadivel, B., Xu, L., et al. (2002). cRaf/MEK/ERK pathway controls protein kinase C-mediated p70S6K activation in adult cardiac muscle cells. The Journal of Biological Chemistry 277(25), 23065-23075.

Isenberg, J. S., Roberts, D. D., \& Frazier, W. A. (2008). CD47: A new target in cardiovascular therapy. Arteriosclerosis, Thrombosis, and Vascular Biology 28(4), 615-621.

Kabir, M. E., Singh, H., Lu, R., Olde, B., Leeb-Lundberg, L. M., \& Bopassa, J. C. (2015). G protein-coupled estrogen receptor 1 mediates acute estrogen-induced cardioprotection via MEK/ERK/GSK-3ß pathway after ischemia/reperfusion. PLoS One 10(9), e0135988.

Kaplan, F. M., Shao, Y., Mayberry, M. M., \& Aplin, A. E. (2011). Hyperactivation of MEKERK1/2 signaling and resistance to apoptosis induced by the oncogenic B-RAF inhibitor, PLX4720, in mutant N-RAS melanoma cells. Oncogene 30(3), 366-371.

Khan, A. Q., Kuttikrishnan, S., Siveen, K. S., Prabhu, K. S., Shanmugakonar, M., Al-Naemi, H. A., et al. (2018). RAS-mediated oncogenic signaling pathways in human malignancies. Seminars in Cancer Biology (pii: S1044-579X(18)30002-6).

Koo, H. M., VanBrocklin, M., McWilliams, M. J., Leppla, S. H., Duesbery, N. S., \& Vande Woude, G. F. (2002). Apoptosis and melanogenesis in human melanoma cells induced by anthrax lethal factor inactivation of mitogen-activated protein kinase kinase. Proceedings of the National Academy of Sciences of the United States of America 99(5), 3052-3057.

Kramann, N., Hasenfuß, G., \& Seidler, T. (2014). B-RAF and its novel negative regulator reticulocalbin 1 (RCN1) modulates cardiomyocyte hypertrophy. Cardiovascular Research 102(1), 88-96.

Kubin, T., Cetinkaya, A., Schönburg, M., Beiras-Fernandez, A., Walther, T., \& Richter, M. (2017). The MEK1 inhibitors UO126 and PD98059 block PDGF-AB induced phosphorylation of threonine 292 in porcine smooth muscle cells. Cytokine 95, 51-54.

Kubin, T., Pöling, J., Kostin, S., Gajawada, P., Hein, S., Rees, W., et al. (2011). Oncostatin M is a major mediator of cardiomyocyte dedifferentiation and remodeling. Cell Stem Cell 9 (5), 420-432.

Lahtinen, R., Kuikka, J., Nousiainen, T., Uusitupa, M., \& Länsimies, E. (1991). Cardiotoxicity of epirubicin and doxorubicin: A double-blind randomized study. European Journal of Haematology 46(5), 301-305.

Larkin, J., Ascierto, P. A., Dréno, B., Atkinson, V., Liszkay, G., Maio, M., et al. (2014). Combined vemurafenib and cobimetinib in BRAF-mutated melanoma. The New England Journal of Medicine 371(20), 1867-1876. 
Li, Z., Jiang, K., Zhu, X., Lin, G., Song, F., Zhao, Y., et al. (2016). Encorafenib (LGX818), a potent BRAF inhibitor, induces senescence accompanied by autophagy in BRAFV600E melanoma cells. Cancer Letters 370(2), 332-344.

Lips, D. J., Bueno, O. F., Wilkins, B. J., Purcell, N. H., Kaiser, R. A., Lorenz, J. N., et al. (2004). MEK1-ERK2 signaling pathway protects myocardium from ischemic injury in vivo. Circulation 109(16), 1938-1941.

Liu, F., Jiang, C. C., Yan, X. G., Tseng, H. Y., Wang, C. Y., Zhang, Y. Y., et al. (2017). BRAF/MEK inhibitors promote CD47 expression that is reversible by ERK inhibition in melanoma. Oncotarget 8(41), 69477-69492.

Long, G. V., Hauschild, A., Santinami, M., Atkinson, V., Mandalà, M., Chiarion-Sileni, V. et al. (2017). Adjuvant Dabrafenib plus Trametinib in stage III BRAF-mutated melanoma. The New England Journal of Medicine 377(19), 1813-1823.

Long, G. V., Stroyakovskiy, D., Gogas, H., Levchenko, E., de Braud, F., Larkin, J., et al. (2014). Combined BRAF and MEK inhibition versus BRAF inhibition alone in melanoma. The New England Journal of Medicine 371(20), 1877-1888.

Long, G. V., Stroyakovskiy, D., Gogas, H., Levchenko, E., de Braud, F., Larkin, J., et al. (2015). Dabrafenib and trametinib versus dabrafenib and placebo for Val600 BRAF-mutant melanoma: A multicentre, double-blind, phase 3 randomised controlled trial. Lancet 386(9992), 444-451.

McArthur, G. A., Chapman, P. B., Robert, C., Larkin, J., Haanen, J. B., Dummer, R., et al (2014). Safety and efficacy of vemurafenib in BRAF(V600E) and BRAF(V600K) mutation-positive melanoma (BRIM-3): Extended follow-up of a phase 3, randomised, open-label study. The Lancet Oncology 15(3), 323-332.

Naitoh, K., Ichikawa, Y., Miura, T., Nakamura, Y., Miki, T., Ikeda, Y., et al. (2006). MitoKATP channel activation suppresses gap junction permeability in the ischemic myocardium by an ERK-dependent mechanism. Cardiovascular Research 70(2), 374-383.

Node, K., Kitakaze, M., Kosaka, H., Minamino, T., Funaya, H., \& Hori, M. (1997). Amelioration of ischemia- and reperfusion-induced myocardial injury by 17beta-estradiol: Role of nitric oxide and calcium-activated potassium channels. Circulation 96(6), 1953-1963.

Planchard, D., Besse, B., Groen, H. J. M., Souquet, P. J., Quoix, E., Baik, C. S., et al. (2016). Dabrafenib plus trametinib in patients with previously treated BRAF(V600E)-mutant metastatic non-small cell lung cancer: An open-label, multicentre phase 2 trial. The Lancet Oncology 17(7), 984-993.

Planchard, D., Smit, E. F., Groen, H. J. M., Mazieres, J., Besse, B., Helland, Å., et al. (2017). Dabrafenib plus trametinib in patients with previously untreated BRAF. The Lancet Oncology 18(10), 1307-1316.

Queirolo, P., Picasso, V., \& Spagnolo, F. (2015). Combined BRAF and MEK inhibition for the treatment of BRAF-mutated metastatic melanoma. Cancer Treatment Reviews 41(6), 519-526.

Robert, C., Karaszewska, B., Schachter, J., Rutkowski, P., Mackiewicz, A., Stroiakovski, D., et al. (2015). Improved overall survival in melanoma with combined dabrafenib and trametinib. The New England Journal of Medicine 372(1), 30-39.

Rose, B. A., Force, T., \& Wang, Y. (2010). Mitogen-activated protein kinase signaling in the heart: Angels versus demons in a heart-breaking tale. Physiological Reviews 90(4), 1507-1546.

Sumimoto, H., Imabayashi, F., Iwata, T., \& Kawakami, Y. (2006). The BRAF-MAPK signaling pathway is essential for cancer-immune evasion in human melanoma cells. The Journal of Experimental Medicine 203(7), 1651-1656.

Zachary, I. (2001). Signaling mechanisms mediating vascular protective actions of vascular endothelial growth factor. American Journal of Physiology. Cell Physiology 280(6), C1375-C1386.

Zhu, H., Tan, L., Li, Y., Li, J., Qiu, M., Li, L., et al. (2017). Increased apoptosis in the paraventricular nucleus mediated by AT1R/Ras/ERK1/2 signaling results in sympathetic hyperactivity and Renovascular hypertension in rats after kidney injury. Frontiers in Physiology 8, 41. 